\title{
Fabrication of flower-like silver nanostructures for rapid detection of caffeine using surface enhanced Raman spectroscopy
}

\author{
Huajun Zheng, ${ }^{a}$ Dejiang Ni, ${ }^{a}$ Zhi Yu, ${ }^{a \dagger}$ Pei Liang ${ }^{b \dagger}$ and Huacai Chen ${ }^{b}$
}

Abstract: Flower-like silver nanoparticles, with diameters of 450 500 nm and protrusions on the surface up to $10 \sim 15 \mathrm{~nm}$, have been successfully synthesized via liquid phase reduction method, by using ascorbic acid as a reductant and polyvinyl pyrrolidone (PVP) as a surfactant. It is found that the proper coating degree of PVP on silver nuclei leads to anisotropic growth of the silver nuclei resulting to the formation of flower-like structure with more protrusions. Furthermore, when the obtained flower-like silver nanostructure is used as Surface Enhanced Raman Scattering(SERS) substrates, SERS signal is still clearly even if the concentration of R6G solution is reduced down to $10^{-8} \mathrm{M}$. The SERS enhancement mechanism of flower-like silver nanostructures in SERS spectroscopy has been further discussed. SERS technology based on the obtained flower-like silver nanostructure substrates can be also used as an effective way to detect caffeine. In this work, it is found that the average intensity of SERS signals at $807 \mathrm{~cm}^{-1}(\mathrm{~N}-\mathrm{C}-\mathrm{H}), 958 \mathrm{~cm}^{-1}$ (Pyrimidine ring) and $1327 \mathrm{~cm}^{-1}$ (Imidazole trigonal ring) linear decreased with the $-\log _{10} \mathrm{C}\left(\mathrm{C}\right.$ is the concentration of caffeine), and the coefficient of determination $\left(\mathrm{R}^{2}\right)$ is as high as 0.975 . This work provides a new way for the quantitative detection of caffeine based on SERS spectroscopy.

Keywords: flower-like silver nanoparticle; liquid phase reduction method; SERS; caffeine

\section{Introduction}

Caffeine is an alkaloid that mainly found in tea, coffee, cocoa, cola nuts and other plants, and is widely used as additive in the field of food, beverage and medicine. The moderate intake of caffeine may improve people's attention and work efficiency, but over intake can cause many unwanted effects.[1] Recent years, many modern techniques have been applied to the analysis of caffeine, such as UV-vis spectrophotometry,[2] Ultrasonic Extracting Technique,[3] High Performance Liquid Chromatography,[4] Near Infrared Spectrum Instrument,[5] etc. However, these techniques are generally expensive, complex and time consuming. Herein, a simple, rapid, sensitive, and low cost analytical technique for analysis of caffeine is very essential.

\footnotetext{
${ }^{a}$ College of Horticulture \& Forestry Sciences, Huazhong Agricultural University, Key Laboratory of Horticultural Plant Biology, Ministry of Education, 430070, Wuhan, China;

${ }^{\mathrm{b}}$ College of Optical and Electronic Technology, China Jiliang University, 310018, Hangzhou, China.

${ }^{\dagger}$ Corresponding author: plianghust@gmail.com (LIANG Pei) and_yuzhi@mail.hzau.edu.cn (YU Zhi)
} 
Recently, Surface Enhanced Raman Scattering (SERS) spectroscopy is widely used in materials analysis, environmental monitoring and other fields.[6] Compared with other techniques, SERS has greater potential for caffeine analysis due to its excellent performance (such as high sensitivity, great flexibility, reproducibility, and reliability).[7] In addition, by combining well-designed microchannels and SERS substrates, more effective and convenient SERS detecting systems had been realized.[8] Bondesson et al. [9]had simulated the Raman spectra of caffeine in the gas phase and aqueous solution by using hybrid density functional theory, and the calculated spectra are correspond well with experimental results. Malgorzata Baranska et al.[10] demonstrated that FT-Raman spectroscopy can be used either for the measurement of an isolated molecule as well as for in situ analyses, and they also used FT-Raman mapping technique to investigate the spatial distribution of caffeine in pharmaceuticals.

It has been reported that the cause of SERS enhancement is mainly due to two mechanisms: electromagnetic effect, caused by localized Surface Plasmon Resonance(LSPR) effect;[11] chemical mechanism, caused by charge transfer effect.[12] Electromagnetic enhancement is the main contribution to drastic enhancement of the signal in SERS and indifferently applies to all analytes.[13] Many researches indicated that the LSPR property is very sensitive to particle size, shape, composition, and arrangement.[14] Currently, many methods, including Hydrothermal method, [15]Liquid phase reduction method,[16] Template method[17] and Nano lithography,[18] have been applied to the fabrication of SERS-active substrates with particular size, shape and so on. In all of the SERS substrates, silver nanostructures shows excellent SERS performance, and various silver nanostructures, such as nanowires,[19] nanorods,[20] nanocubes,[21] and dendritic nanostructures[22] have been used as SERS substrates. Many studies have showed that the controllability, stability, repeatability and uniformity of the substrates play a vital role in SERS detection.[23] Therefore, the preparation of SERS-active substrates with great repeatability, sensitivity and uniformity is highly desirable for broadening the application of SERS detection. 
In this paper, we propose a simple, convenient, reasonable route for the synthesis of SERS-active silver nanostructures, which is carried out in the aqueous medium, produced no heavy metals or organic matter pollution. Silver nanostructures described in this work exhibit high SERS activity and its detection limit for R6G can reach $10^{-8} \mathrm{M}$ or even lower. In addition, the Raman shift, relative intensity, and assignment of characteristic peaks in SERS spectra of caffeine are summarized in this work, which provides the experimental and theoretical basis for the SERS application in qualitative and quantitative detection of caffeine.

\section{Experimental}

\subsection{Materials}

All the chemicals, like caffeine, silver nitrate $\left(\mathrm{AgNO}_{3}\right)$, ascorbic acid, polyvinyl pyrrolidone (PVP), Rhodamine 6G (R6G), were analytical reagents without further purification. Ultrapure water (18.3 M $\Omega$ ) was also used for all solution preparations.

\subsection{Fabrication of Flower-like Silver nanostructures}

A typical synthesis of flower-like silver nanoparticles proceeds was as follows. First, $0.2 \mathrm{ml}$ of $\mathrm{AgNO}_{3}$ solution (1M) and $2 \mathrm{ml}$ of PVP solution (1\%) was both added in $10 \mathrm{ml}$ of ultrapure water. After mixing evenly in the thermostat magnetic stirrer at room temperature, $1 \mathrm{ml}$ of ascorbic acid solution $(0.1$ M) was quickly added into the above mixed solution, and the solution was stirred for next 15 min. Finally, the product of reaction was separated from the solution by centrifugation at $5000 \mathrm{r} / \mathrm{min}$ for $10 \mathrm{~min}$, and it was then dispersed again in $10 \mathrm{ml}$ of ultrapure water, plus these two steps were repeated several times in order to remove impurities.

\subsection{SERS measurement}

Preparation of SERS substrates based on flower-like silver nanoparticles: A certain amount of silver colloid was placed on a Si wafer $(0.5 \times 0.5 \mathrm{~cm})$ and the $\mathrm{Si}$ wafer was transferred to the vacuum oven. Then, the Si wafer was dried at $40^{\circ} \mathrm{C}$ in the vacuum oven for $6 \mathrm{~h}$, to deposit silver nanostructure film on 
the $\mathrm{Si}$ wafer. The as-prepared silver nanostructure films were used as SERS substrate for sensing R6G and caffeine.

SERS measurement: $0.5 \mathrm{ml}$ aliquots of R6G solution with different concentrations ranging from $10^{-8}$ to $10^{-4} \mathrm{M}$ were pipeted onto the SERS substrates, and the solvent evaporated under ambient conditions for SERS measurement. The solutions of caffeine were prepared at different concentrations ranging from $10^{-9}$ to $10^{-4} \mathrm{M}$, and the experiments were carried out using the same procedure as described in the above step. In addition, ten different points on each substrate were selected to detect the molecules in this experiment, to calculate the average Raman spectrum.

\subsection{Characterization}

The structure of the silver nanoparticles were characterized by HITACHI SU8010 field-emission scanning microscopy (resolution: $1.0 \mathrm{~nm}$ ). The EDS spectrum was taken by EDAX TEAM Apollo XL Energy Dispersive Spectrometer (energy resolution: 129 ev). The UV-Visible spectra were taken by Purkinje TU-1901 double-beam spectrophotometer. SERS spectra were recorded by the confocal microscope Raman spectrometer system (Horiba, LabRAM HR) using a $785 \mathrm{~nm}$ laser excitation source with $25 \mathrm{~mW}$ power.

\section{Results and discussion}

\subsection{Characterization of the Flower-like Silver nanostructures}

The obtained silver samples were first characterized by the field emission scanning electron microscopy (SEM) and the energy dispersive spectrum (EDS). Fig.1(A) is the SEM image of the obtained silver nanostructures with large scale, and Fig.1(B) demonstrated the detailed morphology of the silver nanoparticle. As shown in the figures, silver nanoparticles, with diameters of 450 500 nm and protrusions of surface up to $10 \sim 15 \mathrm{~nm}$, have been successfully synthesized, and these nanoparticles are named as flower-like silver nanoparticles in the following article. In addition, the purity of these nanoparticles was confirmed by the EDS spectrum, as shown in Fig.1(C). 
The formation process of silver nanoparticles can be divided into three stages, that is, the induction stage (pre nucleation stage), the nucleation stage and the growth stage. Firstly, a silver cation was reducted to a silver atom upon to the reducting action of reductant, and the silver glomeration was rapidly formed. As the reaction continuing, the number of silver glomeration increases constantly until the nucleation state is reached. After that, the crystal nucleus rapidly generates, and then begins to grow. At the growth stage, if the growth rate of each crystal surface is equal, the growth rate of crystal nucleus will be isotropic and the final particle morphology will be spherical.[24] However, when the surfactant exists, the growth rate of some crystal surfaces will be influenced.[25] PVP is a kind of non-ionic surfactant with excellent properties. Due to its special structure, PVP has different adhesion coefficients on different crystal surfaces.[26] When the PVP molecules preferentially adsorbed on certain crystal surface, the free energy of these crystal surface changes largely compared to crystal surface with no PVP absorption, and further the growth rate of these crystal surface decreases, which can leads to anisotropic growth of the crystal nucleus resulting to the formation of flower-like structure, as shown in the Fig.2.

Fig. 3 is the SEM image of silver nanoparticles obtained in different ascorbic acid addition. Fig. 3(A) shows that, when the ascorbic acid addition is $0.5 \mathrm{ml}$, the particle size of the silver nanoparticles is about 550 600 nm, and the protrusions of the surface are blunt. However, when the amount of ascorbic acid increased to $1 \mathrm{ml}$, as shown in the Fig. 1, the particle size of the silver nanoparticles is about $450 \sim 500 \mathrm{~nm}$, and more and more sharp protrusions appeared on the surface. These phenomena indicate that the ascorbic acid addition has an important influence on the morphology of silver nanoparticles. As the ascorbic acid addition increased to $1 \mathrm{ml}$ from $0.5 \mathrm{ml}$, the more relative amount of reducing agent in the system contained, the faster transformation of silver cation to silver atom was, and the more crystal nucleus were produced in the nucleation stage, which led to the decrease of the molar ratio of PVP and crystal nucleus. Furthermore, the decline of molar ratio indicates the descent of coating degree of PVP on silver nuclei. In this case, the number of exposed crystal surfaces (without surfactant adsorption) is relatively high, which leads to more isotropic growth of the crystal nucleus contributing to the more 
protrusions on the particle surface. Moreover, with the ascorbic acid addition further increase, due to the amount of $\mathrm{AgNO}_{3}$ is enough and other conditions are constant, the production of crystal nucleus will be higher, and the relative content of PVP will be smaller, which leads to a very low coating degree of PVP on the crystal nucleus, and the growth rate of the crystal nucleus is close to the isotropic. As shown in Fig. 3(D), when the ascorbic acid addition increased to $2 \mathrm{ml}$, the surface roughness of the silver nanoparticles decreased obviously. This indicates that the proper coating degree of PVP on silver nuclei is needed in order to form flower-like silver nanoparticles with more sharp protrusions.

\subsection{SERS measurements of R6G on Flower-like Silver nanostructures}

R6G, one of the widely used standard probes for the evaluation of SERS performance was chosen to probe the SERS performance of the obtained flower-like silver nanostructures. A comparison of the SERS activities of the silver nanostructures with different morphologies is shown in Fig. 4. In this measurements, the excitation wavelength is $785 \mathrm{~nm}$, and the signal acquisition time is $1 \mathrm{~s}$. As shown in the Fig. 4(A), the SERS spectra of R6G has a number of peaks within the range of $400 \sim 1800 \mathrm{~cm}^{-1}$, and the pronounced peaks at $771 \mathrm{~cm}^{-1}, 1127 \mathrm{~cm}^{-1}, 1185 \mathrm{~cm}^{-1}, 1311 \mathrm{~cm}^{-1}, 1363 \mathrm{~cm}^{-1}, 1649 \mathrm{~cm}^{-1}, 1508 \mathrm{~cm}^{-1}$ are correspond well with the previous work on R6G.[27] In addition, the synthesized silver nanostructures have great SERS performance, and among them the flower-like silver nanoparticles(the ascorbic acid addition is $1 \mathrm{ml}$ ) exhibit the highest SERS sensitivity.

It is well known that the strong SERS signals generally deriving from the molecules located on particular nanogaps, where the electromagnetic field is largely enhanced, which are called "hot spots".[28] Such "hot spots" support strongly LSPR effect, and thus offer great contribution to SERS. Fig. 4(C) is the UV-Visible absorption spectra of the silver nanoparticles obtained in different ascorbic acid addition. As shown in the Fig. 4(C), the absorption spectra of all silver nanoparticles contained a broad band around $294 \mathrm{~nm}$ due to silver plasmons, and the flower-like silver nanoparticles exhibit the highest intensity, which indicates that it could generated strongest LSPR effect.[29] In the case of SERS "hot spots" in aggregates, maximization of the number of "hot spots" is very valuable.[30] As shown in the SEM image 
(Fig. 1), there are two kinds of nanogaps on the flower-like silver substrate: (1) the nanogap between the two neighbouring silver nanoparticles located on the basement; (2) the nanogap between the two protrusions on the surface of flower-like silver nanoparticle. Obviously, these nanogaps are the locations of SERS "hot spots".

In order to further reveal the SERS ability of the flower-like silver nanoparticles, SERS spectra of different concentrations of R6G solution were measured using the flower-like silver nanoparticles as substrate. Fig. 4(D) shows the SERS spectra of R6G solution with the concentrations increment from $10^{-8}$ to $10^{-4} \mathrm{M}$, and as the concentration of R6G decreases, the SERS signal intensity decreases accordingly. However, the SERS signal can still be observed even when its concentration is reduced down to $10^{-8} \mathrm{M}$, which means that the detection limit for R6G can reach $10^{-8} \mathrm{M}$ or even lower. Fig. 4(E) shows the calibration curve for average intensity of SERS signals at $771 \mathrm{~cm}^{-1}, 1185 \mathrm{~cm}^{-1}$, and $1508 \mathrm{~cm}^{-1}$ as a function of the logarithm of R6G concentration, which the $\mathrm{X}$-axis represents the $-\log _{10} \mathrm{C}$ ( $\mathrm{C}$ is the concentration of R6G) and the Y-axis represents the average intensity of SERS signals. The linear progress equation was $\mathrm{Y}=28920.4-3522.7 \mathrm{X}$, and the coefficient of determination $\left(\mathrm{R}^{2}\right)$ was 0.956 . These results indicated that the flower-like silver structures can be employed as effective SERS substrates for rapid and direct SERS detection.

\subsection{SERS detection of caffeine}

It is well known that the chemical bonds in the molecule have their corresponding vibration frequency, and the vibration frequency is related to the Raman scattering property.[31] By means of analysing the corresponding Raman spectra of the material, the information of material molecules, groups and the chemical bonds in the material can be obtained. Many researches indicated that the group vibration in caffeine molecule can be divided into two categories, namely the stretching vibration and vibration deformation.[32] Stretching vibration is a group of atoms along the chemical bonds of the parallel direction of vibration and leads to chemical bond cycle of elongation or shortening, such as $-\mathrm{CH}_{3}$, $\mathrm{C}=\mathrm{O}, \mathrm{C}=\mathrm{C}, \mathrm{C}-\mathrm{C}, \mathrm{C}-\mathrm{N}$; Deformation vibration is also called bending vibration, which is caused by the 
vibration direction of the atoms in a group and the bond angle, such as $\mathrm{H}-\mathrm{C}=\mathrm{N}, \mathrm{C}-\mathrm{C}, \mathrm{O}=\mathrm{C}-\mathrm{N}$. In general, for a group, the Raman peaks of the stretching vibration is often appear in the higher frequency range of the spectrum, less influenced by the external environment, and the Raman peaks of the deformation vibration are generally in the lower frequency range, which is sensitive to the environmental change.[33] In order to investigate the effect of silver nanoparticles on the Raman scattering of caffeine molecules, the normal Raman spectra and SERS spectra of caffeine are compared in Fig.5. Fig.5(A) is the normal Raman spectroscopy of pure caffeine powder, and Fig.5(B) is the SERS spectra of caffeine solution $\left(10^{-4}\right.$ M) adsorbed on the flower-like silver nanoparticles. To compare and analyse the difference of the spectral curves of the two spectra, as shown in Table 1, we sum up the Raman shifts and relative intensities of the characteristic peaks at the two spectra, and identify the vibrational assignment of the peaks by consulting the literature.[34, 35]

As the Fig.5 shows, the SERS spectra from caffeine molecules adsorbed on the flower-like silver nanostructures were significantly different from the normal Raman spectra of caffeine in many aspects, including the number of peaks and the relative intensities, which could show that there are strong interactions between caffeine molecules and silver nanostructures. The electromagnetic effect is a physical mechanism and the nature of it is the surface plasmon resonance of exciting light and metallic nanoparticles. In this case, the pattern of the SERS spectra is basically unchanged compared to conventional Raman spectra. However, the charge transfer effect is a chemical mechanism, which involves specific interactions between analytes molecules and the metal particles, and is not related to the SERS itself but to the modification of the adsorbate polarizability.[36] In comparison with electromagnetic enhancement, chemical mechanism may contributes to a lesser extent to the total SERS enhancement, but it will significantly influence the pattern of the SERS spectra.[37] As shown in the Table 1, the Raman signals originate from $\mathrm{O}=\mathrm{C}-\mathrm{N}$ deformation, $\mathrm{N}-\mathrm{C}-\mathrm{H}$ deformation, Pyrimidine ring deformation, C-C deformation, $\mathrm{C}-\mathrm{N}$ stretching, and Imidazole trigonal ring stretching are significantly enhanced, and their frequency shifts are very slight. Meanwhile, for some vibrations, such as $\mathrm{O}=\mathrm{C}-\mathrm{N}$ 
deformation, $\mathrm{O}=\mathrm{C}-\mathrm{C}$ deformation, and $\mathrm{C}-\mathrm{N}$ stretching, their Raman signal intensities are not obviously enhanced or even disappear. Hence, it is possible that both electromagnetic and chemical mechanisms are involved in the interaction between caffeine molecules and silver nanoparticles, but the contribution of the electromagnetic mechanism is dominant.

Fig. 6(A) shows the SERS spectra of different concentrations of caffeine solution using the flowerlike silver nanostructures as substrate. In this measurements, the excitation wavelength is $785 \mathrm{~nm}$, and the signal acquisition time is $4 \mathrm{~s}$. It is found that, as the concentration of caffeine decreases, the SERS signal intensity decreases accordingly. However, even if the concentration of caffeine decreased to $10^{-9} \mathrm{M}$, the SERS signal of caffeine at $807 \mathrm{~cm}^{-1}, 958 \mathrm{~cm}^{-1}, 1040 \mathrm{~cm}^{-1}$, and $1327 \mathrm{~cm}^{-1}$ still can be distinctly observed, demonstrating the high sensitivity of the flower-like silver nanostructures for SERS detection of caffeine. In addition, it is found that the SERS of caffeine is more sensitive than SERS of R6G. Fig.6(C) shows the UV-Visible absorption spectrum of caffeine. As shown in the spectrum, no obvious peak present at 785 $\mathrm{nm}$, indicating caffeine does not have optical resonance at excitation laser wavelength of $785 \mathrm{~nm}$, which further reveal that charge transfer effect may be involved in the strong SERS of caffeine.

Fig. 6(B) shows the calibration curve for average intensity of SERS signals at $807 \mathrm{~cm}^{-1}, 958 \mathrm{~cm}^{-1}$, and $1327 \mathrm{~cm}^{-1}$ as a function of the logarithm of caffeine concentration, which the $\mathrm{X}$-axis represents the $\log _{10} \mathrm{C}(\mathrm{C}$ is the concentration of caffeine) and the $\mathrm{Y}$-axis represents the average intensity of SERS signal. It was found that the average intensity of SERS signals linear decreased with the $-\log _{10} \mathrm{C}$, and the coefficient of determination $\left(\mathrm{R}^{2}\right)$ is as high as 0.975 . The linear progress equation was $\mathrm{Y}=7513.7-809 \mathrm{X}$. This phenomenon provides a basis for the quantitative detection of caffeine based on SERS spectroscopy.

\section{Conclusions}

In summary, we have developed a simple and facile approach for the preparation of flowerlike silver nanostructures, which can serve as an effective SERS detection platform for caffeine. The possible mechanism for the formation of flower-like silver nanostructures is also explained 
based on a series of experiments. It was found that, when the other conditions are constant, the addition of reductant in the system can control the coating degree of surfactant on crystal nucleus, which have great influence on the morphology of nanostructure. The SERS measurements showed that the flower-like silver nanostructures have a high SERS activity, and the SERS performance is better than that of spherical and polyhedral silver nanoparticles, which indicated that the flower-like silver nanostructures can provide more "hot spots" than a regular structure due to the unique flower-like nanostructures. Furthermore, the detection sensitivity of $10^{-8} \mathrm{M}$ based on the flower-like silver nanostructures can be reached for R6G molecules.

In addition, this work investigates the SERS spectra of caffeine based on the flower-like silver nanostructures. It was found that, the contribution of the electromagnetic mechanism is dominant for SERS performance, and even if the concentrations of caffeine decreased to $10^{-9} \mathrm{M}$, the SERS signal at $807 \mathrm{~cm}^{-1}, 958 \mathrm{~cm}^{-1}, 1040 \mathrm{~cm}^{-1}$, and $1327 \mathrm{~cm}^{-1}$ still can be distinctly observed in the SERS spectra, which means that the detection limit of caffeine can reach $10^{-9} \mathrm{M}$. Besides, the average intensity of SERS signals at $807 \mathrm{~cm}^{-1}, 958 \mathrm{~cm}^{-1}$, and $1327 \mathrm{~cm}^{-1}$ linear decreased with the $-\log _{10} \mathrm{C}$, and the coefficient of determination $\left(\mathrm{R}^{2}\right)$ is as high as 0.975 , which indicates that the flower-like silver nanostructures have great potentials as effective SERS substrates for trace detection and monitoring of caffeine.

\section{Acknowledgements}

The Project was supported by the National Science Foundation for Young Scholars of China (Grant No.31000316). The Project was supported by Application Research Program of Commonweal Technology of Zhejiang Province (No. 2014C37042).

\section{References}

[1] P. Nawrot, S. Jordan, J. Eastwood, J. Rotstein, A. Hugenholtz and M. Feeley, Effects of caffeine on human health, Food Additives \& Contaminants, 20 (2003) 1-30. 
[2] X. Wen, Q. Yang, Z. Yan and Q. Deng, Determination of cadmium and copper in water and food samples by dispersive liquid-liquid microextraction combined with UV-vis spectrophotometry, Microchemical Journal, 97 (2011) $249-254$.

[3] H.R. Liu and W.M. Dong, Determination of Caffeine in Tea by Ultrasonic Extracting Technique, Food Research \& Development, (2013).

[4] J.L. Blauch and S.M. Tarka, HPLC Determination of Caffeine and Theobromine in Coffee, Tea, and Instant Hot Cocoa Mixes, Journal of Food Science, 48 (1983) 745-747.

[5] P. C, E.-D. I, G.-S. JM and F. M, Use of near-infrared spectroscopy and feature selection techniques for predicting the caffeine content and roasting color in roasted coffees, J.agric.food Chem, 55 (2007) 7477-7488.

[6] S. Sebastian, Surface-Enhanced Raman Spectroscopy: Concepts and Chemical Applications, Angewandte Chemie : international Ed, 53 (2014) 4756-4795.

[7] D. Cialla, A. M01rz, R. B02hme, F. Theil, K. Weber, M. Schmitt and J. Popp, Surface-enhanced Raman spectroscopy (SERS): progress and trends, Analytical \& Bioanalytical Chemistry, 403 (2012) 27-54.

[8] Q. Zhou and T. Kim, Review of microfluidic approaches for surface-enhanced Raman scattering, Sensors and Actuators B: Chemical, 227 (2016) 504-514.

[9] L. Bondesson, K.V. Mikkelsen, Y. Luo, P. Garberg, Hans, Aring and gren, Hydrogen bonding effects on infrared and Raman spectra of drug molecules, Spectrochim Acta A Mol Biomol Spectrosc, 66 (2007) 213-224.

[10] M. Baranska and L.M. Proniewicz, Raman mapping of caffeine alkaloid, Vibrational Spectroscopy, 48 (2008) 153157.

[11] Y.S. Yamamoto, Y. Ozaki and T. Itoh, Recent progress and frontiers in the electromagnetic mechanism of surfaceenhanced Raman scattering, Journal of Photochemistry \& Photobiology C Photochemistry Reviews, (2014) 81-104.

[12] Y.F. Huang, D.Y. Wu, H.P. Zhu, L.B. Zhao, G.K. Liu, B. Ren and T. ZQ, Surface-enhanced Raman spectroscopic study of p-aminothiophenol, Physical Chemistry Chemical Physics, 14 (2012) 8485-8497.

[13] A. Hakonen, P.O. Andersson, M.S. Schmidt, T. Rindzevicius and M. K011l, Explosive and chemical threat detection by surface-enhanced Raman scattering: A review, Analytica Chimica Acta, (2015).

[14] Y. Xia, X. Xia and H.C. Peng, Shape-Controlled Synthesis of Colloidal Metal Nanocrystals: Thermodynamic versus Kinetic Products, Journal of the American Chemical Society, (2015). 
[15] D. Li, S. Wu, Q. Wang, Y. Wu, W. Peng and L. Pan, Ag@C Core-Shell Colloidal Nanoparticles Prepared by the Hydrothermal Route and the Low Temperature Heating-Stirring Method and Their Application in Surface Enhanced Raman Scattering, Journal of Physical Chemistry C, 116 (2012) 12283-12294.

[16] M.M. Alam, J. Wei, H.N. Luitel, Y. Ozaki, T. Watari and K. Nakashima, Template Free Synthesis of Dendritic Silver Nanostructure and Its Application to Surface-Enhanced Raman Scattering, Rsc Advances, (2014).

[17] L. Y, G. J and Y. Y, Templated synthesis of nanostructured materials, Chemical Society Reviews, 42 (2013) 26102653.

[18] M. Chirumamilla, G. Das, A. Toma, A. Gopalakrishnan, R.P. Zaccaria, C. Liberale, F.D. Angelis and E.D. Fabrizio, Optimization and characterization of $\mathrm{Au}$ cuboid nanostructures as a SERS device for sensing applications, Microelectronic Engineering, 97 (2012) 189-192.

[19] M. Chen, C. Wang, X. Wei and G. Diao, Rapid Synthesis of Silver Nanowires and Network Structures under Cuprous Oxide Nanospheres and Application in Surface-Enhanced Raman Scattering, Journal of Physical Chemistry C, 117 (2013) 13593-13601.

[20] S. Mohapatra, S. Siddhanta, D.R. Kumar, C. Narayana and T.K. Maji, Facile and Green Synthesis of SERS Active and Ferromagnetic Silver Nanorods, European Journal of Inorganic Chemistry, 2010 (2010) 4969-4974.

[21] T. Zhang, L. Zhang, S. Yang, Z. Yang and B. Ding, SERS Effect of Silver Nanocubes Substrates with Two Different Morphologies, Rare Metal Materials \& Engineering, 36 (2007) 1844-1847.

[22] Q. Wang, d. wu and z. chen, Ag dendritic nanostructures for rapid detection of thiram based on surface-enhanced Raman scattering, RSC Adv., (2015).

[23] G. Luca and G. Duncan, Molecularly-mediated assemblies of plasmonic nanoparticles for Surface-Enhanced Raman Spectroscopy applications, Chem.soc.rev, 41 (2012) 7085-7107.

[24] S. Yugang and X. Younan, Shape-Controlled Synthesis of Gold and Silver Nanoparticles, Science, 298 (2003) págs. 2176-2179.

[25] W. Wang, S. Efrima and O. Regev, Directing Silver Nanoparticles into Colloid61Surfactant Lyotropic Lamellar Systems, Journal of Physical Chemistry B, 103 (1999).

[26] F. Rasmus, O. Ping, H. Mads, D. Duy Anh, H. Hans Jürgen and A. Herman, PVP-coated silver nanoparticles and silver ions induce reactive oxygen species, apoptosis and necrosis in THP-1 monocytes, Toxicology Letters, 190 (2009) 156-162. 
[27] A.M. Michaels, M. Nirmal and L.E. Brus, Surface Enhanced Raman Spectroscopy of Individual Rhodamine 6G Molecules on Large Ag Nanocrystals, Journal of the American Chemical Society, 121 (1999) 9932-9939.

[28] M. Rycenga, X. Xia, C.H. Moran, F. Zhou, D. Qin, Z.Y. Li and Y. Xia, Generation of hot spots with silver nanocubes for single-molecule detection by surface-enhanced Raman Scattering (EI), Angewandte Chemie International Edition, 50 (2011).

[29] T. Tan, C. Tian, Z. Ren, J. Yang, Y. Chen, L. Sun, Z. Li, A. Wu, J. Yin and H. Fu, LSPR-dependent SERS performance of silver nanoplates with highly stable and broad tunable LSPRs prepared through an improved seedmediated strategy, Physical Chemistry Chemical Physics Pccp, 15 (2013) 21034-21042.

[30] Y.S. Yamamoto, K. Hasegawa, Y. Hasegawa, N. Takahashi, Y. Kitahama, S. Fukuoka, N. Murase, Y. Baba, Y. Ozaki and T. Itoh, Direct conversion of silver complexes to nanoscale hexagonal columns on a copper alloy for plasmonic applications, Physical chemistry chemical physics : PCCP, 15 (2013) 14611-14615.

[31] A. Campion and P. Kambhampati, Surface-enhanced Raman scattering, Chem.soc.rev, 27 (1998) 241-250.

[32] S.K. Srivastava and V.B. Singh, Ab initio and DFT studies of the structure and vibrational spectra of anhydrous caffeine, Spectrochimica Acta Part A Molecular \& Biomolecular Spectroscopy, 115 (2013) 45-50.

[33] M.D. Matas, H.G.M. Edwards, E.E. Lawson, L. Shields and P. York, FT-Raman spectroscopic investigation of a pseudopolymorphic transition in caffeine hydrate, Journal of Molecular Structure, 440 (1998) 97-104.

[34] S. Gunasekaran, G. Sankari and S. Ponnusamy, Vibrational spectral investigation on xanthine and its derivativestheophylline, caffeine and theobromine, Spectrochimica Acta Part A Molecular \& Biomolecular Spectroscopy, 61 (2005) $117-127$.

[35] X. Chen, H. Gu, G. Shen, X. Dong and J. Kang, Spectroscopic study of surface enhanced Raman scattering of caffeine on borohydride-reduced silver colloids, Journal of Molecular Structure, 975 (2010) 63-68.

[36] E.C.L. Ru, E. Blackie, M. Meyer and P.G. Etchegoin, Surface enhanced Raman scattering enhancement factors: a comprehensive study, in: J. Phys. Chem.-US C, 2007, pp. 13794-13803.

[37] J.E. Moore, S.M. Morton and L. Jensen, Importance of Correctly Describing Charge-Transfer Excitations for Understanding the Chemical Effect in SERS, J.phys.chem.lett, 3 (2012) 2470-2475. 


\section{Table list:}

Table 1 The main peaks of caffeine Raman spectra and SERS spectra within the range of $500 \sim 1700 \mathrm{~cm}^{-1}$ and their vibrational assignment. 
Table 1

\begin{tabular}{|c|c|c|}
\hline Raman spectra $\left(\mathrm{cm}^{-1}\right)$ & SERS spectra $\left(\mathrm{cm}^{-1}\right)$ & Vibrational assignment \\
\hline 556 vs & $607 \mathrm{~m}$ & $\mathrm{O}=\mathrm{C}-\mathrm{N}$ deformation \\
\hline $644 \mathrm{~s}$ & $658 \mathrm{~m}$ & $\mathrm{O}=\mathrm{C}-\mathrm{N}$ deformation \\
\hline $741 \mathrm{~s}$ & - & $\mathrm{O}=\mathrm{C}-\mathrm{C}$ deformation \\
\hline $801 \mathrm{~s}$ & 807 vs & $\mathrm{N}-\mathrm{C}-\mathrm{H}$ deformation \\
\hline $928 \mathrm{~m}$ & - & Imidazole ring deformation \\
\hline $974 \mathrm{w}$ & $958 \mathrm{~s}$ & Pyrimidine ring deformation \\
\hline $1020 \mathrm{~m}$ & 1040 vs & C-C deformation \\
\hline $1239 \mathrm{~m}$ & - & $\mathrm{C}-\mathrm{N}$ stretching \\
\hline $1284 \mathrm{~m}$ & $1269 \mathrm{w}$ & $\mathrm{C}-\mathrm{N}$ stretching \\
\hline 1328 vs & $1327 \mathrm{~m}$ & Imidazole trigonal ring stretching \\
\hline $1410 \mathrm{w}$ & $1415 \mathrm{w}$ & $\mathrm{C}-\mathrm{N}$ stretching \\
\hline $1601 \mathrm{w}$ & $1593 \mathrm{w}$ & $\mathrm{C}=\mathrm{C} \quad$ stretching \\
\hline
\end{tabular}

vs=very strong; $\quad$ s=strong; $\quad \mathbf{m}=$ =middle; $\quad$ w=weak 


\section{Figure list:}

Fig. 1 (A, B) SEM image and (C) EDS spectrum of the flower-like silver nanoparticles (the ascorbic acid addition is $1 \mathrm{ml})$.

Fig. 2 Schematic illustration for the formation of the flower-like silver nanoparticle.

Fig. 3 SEM image of silver nanoparticles obtained in different ascorbic acid addition: (A, B) $0.5 \mathrm{ml} ;(\mathrm{C}, \mathrm{D}) 2 \mathrm{ml}$.

Fig. 4 (A) SERS spectra and (B) SERS intensity at $611 \mathrm{~cm}^{-1}$ of R6G $\left(10^{-4} \mathrm{M}\right)$ adsorbed on the Si wafer (a) and silver nanoparticles prepared with different volume of ascorbic acid solution: (b)0.5 $\mathrm{ml}$; (c)1 ml; (d)2 ml; (C) UV-Visible spectra of the silver nanoparticles obtained in different ascorbic acid addition: (b) $0.5 \mathrm{ml}$; (c)1 ml; (d)2 ml; (D) SERS spectra of R6G with different concentrations adsorbed on the flower-like silver nanoparticles: (a) $10^{-4}$, (b) $10^{-5}$, (c) $10^{-6}$, (d) $10^{-7}$, (e) $10^{-8}$ M. (E) The average intensity of SERS signals at $771 \mathrm{~cm}^{-1}, 1185 \mathrm{~cm}^{-1}$, and $1508 \mathrm{~cm}^{-1}$ as a function of the logarithm of $\mathrm{R} 6 \mathrm{G}$ concentration.

Fig. 5 (A) The normal Raman spectroscopy of caffeine powder; $\left(\mathrm{A}_{\text {in }}\right)$ The molecular structure of caffeine; (B) SERS spectra of caffeine $\left(10^{-4} \mathrm{M}\right)$ adsorbed on the flower-like silver nanoparticles.

Fig. 6 (A) SERS spectra of caffeine with different concentrations adsorbed on the flower-like silver nanoparticles: (a) $10^{-4}$, (b) $10^{-5}$, (c) $10^{-6}$, (d) $10^{-7}$, (e) $10^{-8}$, (f) $10^{-9} \mathrm{M}$; (B) The average intensity of SERS signals at $807 \mathrm{~cm}^{-1}, 958 \mathrm{~cm}^{-1}$, and $1327 \mathrm{~cm}^{-1}$ as a function of the logarithm of caffeine concentration. (C) The UV-Visible absorption spectrum of caffeine. 

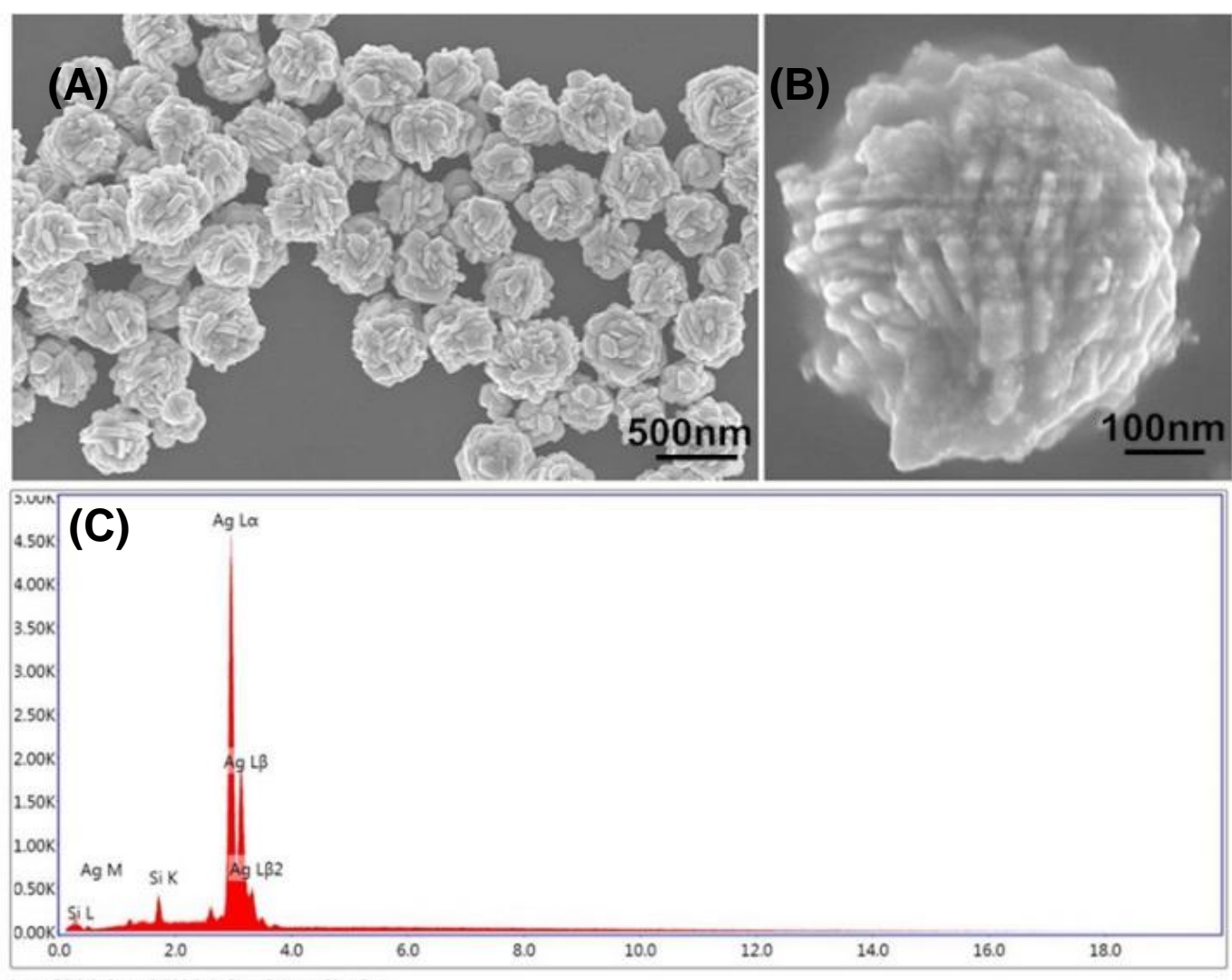

Lsec: 50.00 Cnts 0.000 keV Det: Octane Plus Det

Fig. 1 


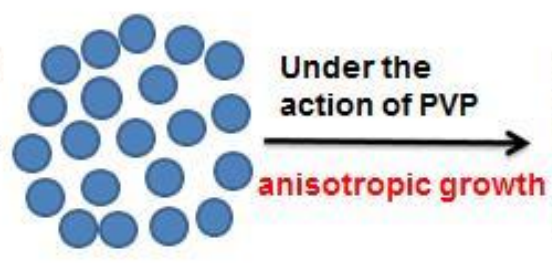

$\mathrm{Ag}^{0}$ glomeration

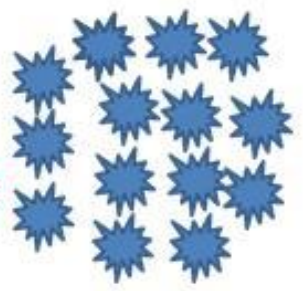

Flower-like

Ag nanoparticle

Fig.2 


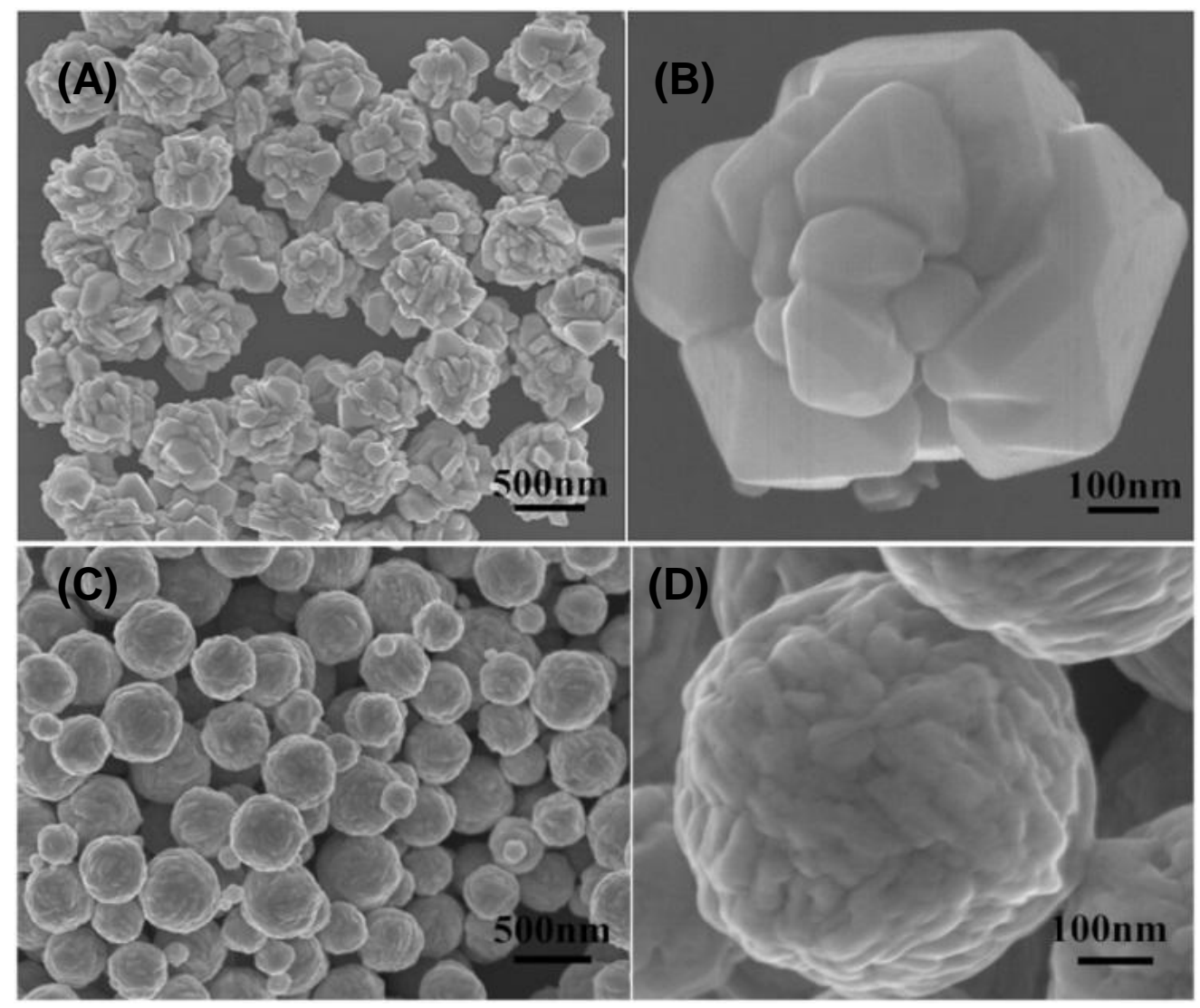

Fig. 3 

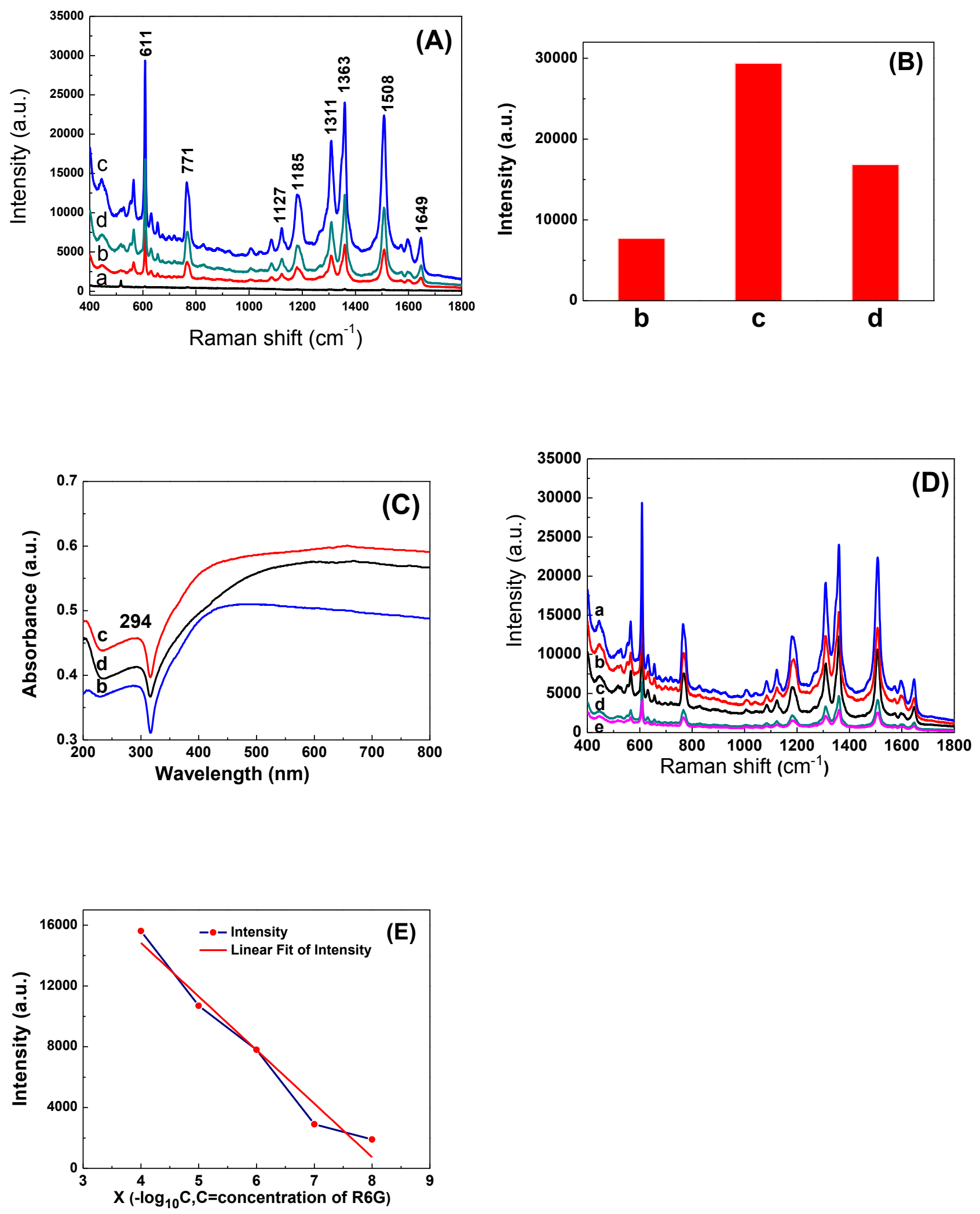

Fig. 4 

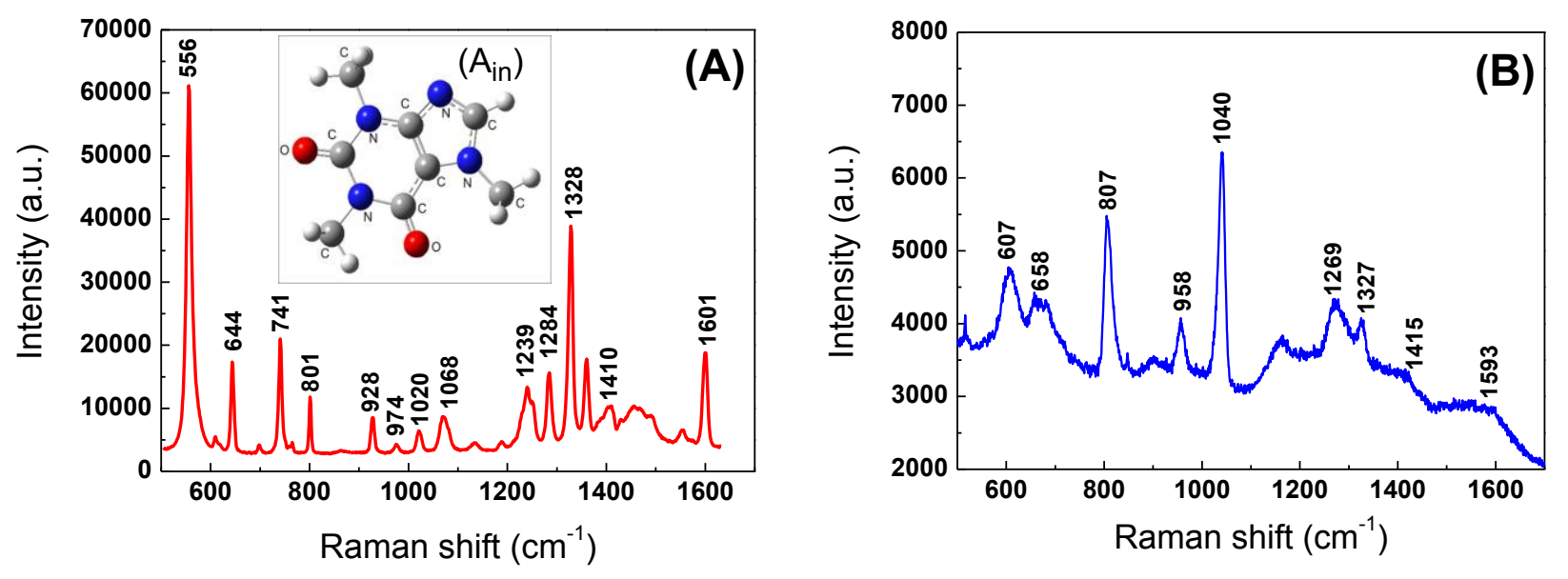

Fig.5 

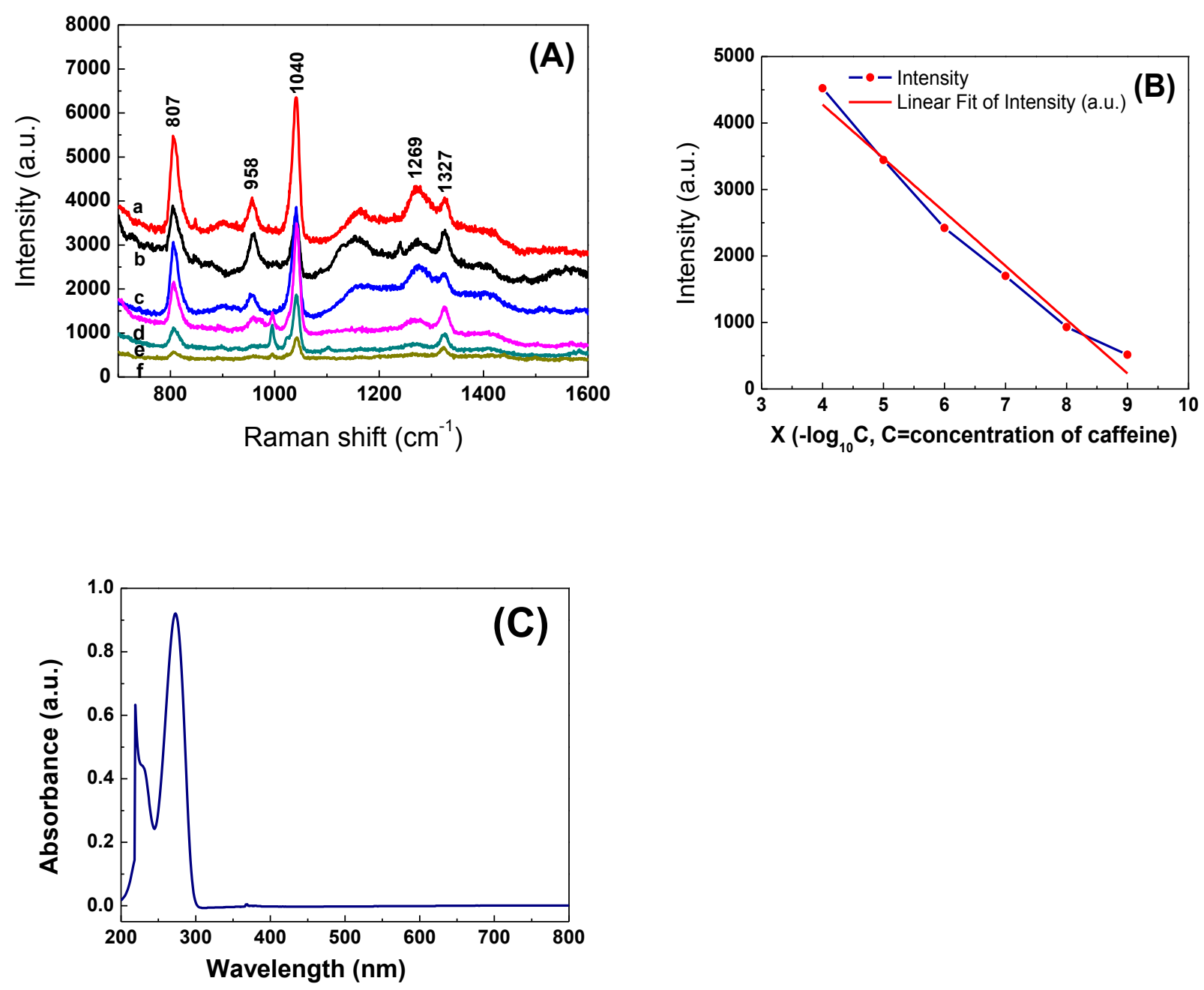

Fig. 6 